\title{
Wigner functions for the pair angle and orbital angular momentum
}

\author{
H. A. Kastrup* \\ DESY, Theory Group, Notkestrasse 85, D-22607 Hamburg, Germany
}

(Received 8 January 2016; published 15 December 2016)

\begin{abstract}
The problem of constructing physically and mathematically well-defined Wigner functions for the canonical pair angle $\theta$ and angular momentum $p$ is solved. While a key element for the construction of Wigner functions for the planar phase space $\left\{(q, p) \in \mathbb{R}^{2}\right\}$ is the Heisenberg-Weyl group, the corresponding group for the cylindrical phase space $\left\{(\theta, p) \in S^{1} \times \mathbb{R}\right\}$ is the Euclidean group $E(2)$ of the plane and its unitary representations. Here the angle $\theta$ is replaced by the pair $(\cos \theta, \sin \theta)$, which corresponds uniquely to the points on the unit circle. The main structural properties of the Wigner functions for the planar and the cylindrical phase spaces are strikingly similar. A crucial role is played by the sinc function, which provides the interpolation for the discontinuous quantized angular momenta in terms of the continuous classical ones, in accordance with the famous Whittaker cardinal function well known from interpolation and sampling theories. The quantum mechanical marginal distributions for the angle (continuous) and angular momentum (discontinuous) are, as usual, uniquely obtained by appropriate integrations of the $(\theta, p)$ Wigner function. Among the examples discussed is an elementary system of simple cat states.
\end{abstract}

DOI: 10.1103/PhysRevA.94.062113

\section{INTRODUCTION}

In 1932 Wigner introduced a function in the classical $(q, p)$ phase space of a given system with the aim of describing the quantum mechanical statistical properties of that system partially in terms of classical ones [1,2]. After a slow start the concept has become quite popular and efficient, e.g., in quantum optics [3-7] and time-frequency analysis [8,9].

In view of the successes of Wigner functions on the topologically trivial planar phase space $\mathbb{R}^{2}$, attempts have been made to generalize the concept to other phase spaces, in particular, to that of a simple rotator around a fixed axis, its position given by an angle $\theta$ and its angular momentum by a real number $p$, thus having a phase space corresponding topologically to a cylinder of infinite length, i.e., $S^{1} \times \mathbb{R}$.

A considerable obstacle for the quantum theory of that space is the treatment of the angle which has no satisfactory self-adjoint operator counterpart quantum mechanically [10]. Nevertheless a "Hermitian" angle operator was introduced formally, then exponentiated to a seemingly unitary one, and the classically continuous angular momentum $p$ was replaced by the discrete quantum mechanical $l \in \mathbb{Z}$ one, thus quantizing a hybrid "phase space" $S^{1} \times \mathbb{Z}$. This approach was started by Berry [11] and Mukunda [12] and has since been pursued by quite a number of authors. More recent typical examples are Refs. [13-15].

The present paper provides consistent Wigner functions in the classical physical phase space $S^{1} \times \mathbb{R}$ including a mathematically satisfactory treatment of the angle $\theta \in \mathbb{S}^{1}$. We see below (Sec. II B) where the "hybrid" case $S^{1} \times \mathbb{Z}$ is to be placed within the framework proposed here.

Parallel to these attempts to find Wigner functions for systems on a cylinder its quantum mechanics was surprisingly described-in two different approaches-in terms of unitary representations of the Euclidean group E(2) of the plane. Fronsdal initiated the first of these two in the framework of the

\footnotetext{
*hans.kastrup@desy.de
}

so-called Moyal or $\star$ quantization [16], followed by a number of papers by other authors, e.g., [17] and [18].

In a different approach Isham discussed very convincingly [19] the group theoretical quantization of the phase space $S^{1} \times \mathbb{R}$ in terms of irreducible unitary representations of $E(2)$ and its covering groups. His results were used in order to discuss in detail the appropriate quantum mechanics of the $(\theta, p)$ rotator [10].

The present paper is a continuation of Ref. [10]. It provides a mathematically and physically consistent Wigner function on a cylindrical phase space by utilizing the tools discussed in Ref. [10]: Representing the angle $\theta$ of the rotator by the equivalent pair $(\cos \theta, \sin \theta)$ introduces-together with the angular momentum $p$-simultaneously the three-dimensional Lie algebra of the Euclidean group $E(2)$ in two dimensions. Unitary representations of that group provide the appropriate quantum mechanics of the rotator. Using slightly modified ideas for constructing "Wigner operators" from the Lie algebra of associated groups by Wolf and collaborators [20-22] leads to the corresponding operator $V(\theta, p)$ in terms of the Lie algebra of $E(2)$ (Sec. II). Wigner functions are obtained by calculating matrix elements of that operator within an irreducible representation of $E(2)$. Matrix elements between two different states yield Wigner-Moyal functions [23], whereas two identical states provide Wigner functions proper [1], the latter being a special case of the former.

The resulting structures and properties (Sec. III) have a very remarkable resemblance to those of the well-known $(q, p)$ case (for reviews see, e.g., Refs. [2], [24], and [25]). An essential feature of the $(\theta, p)$ Wigner-Moyal functions is the central role played by the sinc function (sinus cardinalis),

$$
\operatorname{sinc} \pi(p-m)=\frac{\sin \pi(p-m)}{\pi(p-m)}, \quad p \in \mathbb{R}, \quad m \in \mathbb{Z},
$$

which interpolates between the discontinuous quantized angular momenta $m(\hbar=1)$ by means of the continuous classical ones $p$ [for a graph of function (1), see Fig. 1 in Sec. IV C].

In 1915 E. T. Whittaker introduced his-now famouscardinal function [26] for the continuous interpolation of 
functions for which discrete values are known by using functions (1). Since then these functions have been playing an increasingly important role in the fields of interpolation, sampling, and signal processing theories (see the reviews in [27-34].

The time evolution of Wigner-Moyal functions is determined by the Hermitian operator $K(\theta, p)=i[H, V(\theta, p)]$ (Sec. III C).

The quantum mechanical marginal distributions $|\psi(\theta)|^{2}$ and $b_{m}=\left|c_{m}\right|^{2}$, where the $c_{m}$ are the expansion coefficients of the wave function $\psi(\varphi)$ with respect to the basis $e_{m}(\varphi)=$ $\exp (\operatorname{im} \varphi), m \in \mathbb{Z}$, are obtained-as usual-by integrating the Wigner function $V_{\psi}(\theta, p)$ over $p$ and $\theta$, respectively. The latter integration yields a cardinal function $\omega_{\psi}(p)$ from which the probabilities $b_{m}$ can be extracted by means of the orthonormality of the sinc functions, (1) (Sec. IV B).

Four examples of Wigner functions for certain typical states are discussed in Sec. IV C: that of the basis function $e_{m}(\varphi)$, that of the cat state $\left(e_{1}(\varphi)+e_{-1}(\varphi)\right) / \sqrt{2}$, that of "minimal uncertainty" states $\psi_{e}(\varphi)$, which lead to the von Mises statistical distribution $\left|\psi_{e}(\varphi)\right|^{2}=\exp (2 s \cos \varphi) / I_{0}(2 s)$ and which are the analog of the Gaussian wave packets in the $(q, p)$ case, and, finally, that of thermal states associated with the Hamiltonian $H=\varepsilon L^{2}$, where $L$ is the quantum mechanical counterpart of the classical angular momentum $p$.

\section{THE AUXILIARY ROLE OF THE EUCLIDEAN GROUP E(2)}

The close relationship between the rotator and group $E$ (2) comes about as follows [10]: In order to avoid the problems with a quantization of the angle $\theta$ itself Louisell and Mackey suggested, independently in 1963 [35,36], using the pair $(\cos \theta, \sin \theta)$ instead, because it is in one-to-one correspondence to the points on the unit circle and it consists of smooth-bounded $2 \pi$-periodic functions the quantization of which appears most likely to be easier to handle than that for the angle itself.

\section{A. Classical E(2) group theory}

The basic functions

$$
\tilde{h}_{1}=\cos \theta, \quad \tilde{h}_{2}=\sin \theta, \quad \tilde{h}_{3}=p_{\theta} \equiv p
$$

in the classical phase space

$$
\mathcal{S}_{\theta, p}=\{s=(\theta, p) ; \theta \in \mathbb{R} \bmod 2 \pi, p \in \mathbb{R}\}
$$

obey the Poisson brackets

$$
\left\{\tilde{h}_{3}, \tilde{h}_{1}\right\}_{\theta, p}=\tilde{h}_{2},\left\{\tilde{h}_{3}, \tilde{h}_{2}\right\}_{\theta, p}=-\tilde{h}_{1},\left\{\tilde{h}_{1}, \tilde{h}_{2}\right\}_{\theta, p}=0 .
$$

They constitute the Lie algebra of the three-parametric Euclidean group $E(2)=\{g(\vartheta, \vec{a})\}$ of the plane $\vec{x} \rightarrow R(\vartheta)$. $\vec{x}+\vec{a}$, i.e.,

$$
g(\vartheta, \vec{a}) \circ \vec{x}=\left(\begin{array}{cc}
\cos \vartheta & -\sin \vartheta \\
\sin \vartheta & \cos \vartheta
\end{array}\right)\left(\begin{array}{l}
x_{1} \\
x_{2}
\end{array}\right)+\left(\begin{array}{l}
a_{1} \\
a_{2}
\end{array}\right) .
$$

We write

$$
\left(\begin{array}{cc}
\cos \vartheta & -\sin \vartheta \\
\sin \vartheta & \cos \vartheta
\end{array}\right)\left(\begin{array}{l}
x_{1} \\
x_{2}
\end{array}\right) \equiv R(\vartheta) \vec{x} \equiv \vec{x}_{\vartheta}
$$

In the following it is advantageous to cast relations (5) into those of $3 \times 3$ matrices [37], for which group multiplication, etc., are implemented by matrix multiplication, etc.,

$$
g(\vartheta, \vec{a}) \circ\left(\begin{array}{c}
x_{1} \\
x_{2} \\
1
\end{array}\right)=\left(\begin{array}{ccc}
\cos \vartheta & -\sin \vartheta & a_{1} \\
\sin \vartheta & \cos \vartheta & a_{2} \\
0 & 0 & 1
\end{array}\right)\left(\begin{array}{c}
x_{1} \\
x_{2} \\
1
\end{array}\right)
$$

from which one can read off immediately the Lie algebra generators

$$
\begin{aligned}
\tilde{L} & =\left(\begin{array}{rrr}
0 & -1 & 0 \\
1 & 0 & 0 \\
0 & 0 & 0
\end{array}\right), \quad \tilde{K}_{1}=\left(\begin{array}{lll}
0 & 0 & 1 \\
0 & 0 & 0 \\
0 & 0 & 0
\end{array}\right) \\
\tilde{K}_{2} & =\left(\begin{array}{lll}
0 & 0 & 0 \\
0 & 0 & 1 \\
0 & 0 & 0
\end{array}\right) .
\end{aligned}
$$

They obey the commutation relations

$$
\left[\tilde{L}, \tilde{K}_{1}\right]=\tilde{K}_{2}, \quad\left[\tilde{L}, \tilde{K}_{2}\right]=-\tilde{K}_{1}, \quad\left[\tilde{K}_{1}, \tilde{K}_{2}\right]=0
$$

These are obviously isomorphic to those of Eq. (4). In the corresponding quantum theory the generators $\tilde{L}$ and $\overrightarrow{\tilde{K}}=\left(\tilde{K}_{1}, \tilde{K}_{2}\right)$ will turn into the self-adjoint operators $L$ and $\vec{K}$, which describe the quantum mecchanics of the angular momentum $p$ and the pair $(\cos \theta, \sin \theta)$.

As for the latter there is the following subtlety: In Eq. (9) the generators $\tilde{K}_{j}$ are not normalized. If one multiplies them with a nonzero constant, the new $\tilde{K}_{j}$ obey the same communitation relations as the old ones. This means that the relevant classical observable here is a direction, a vector (e.g., $\in \mathbb{C}$ ), or a half-ray

$$
\vec{\chi}(\theta)=\chi(\cos \theta, \sin \theta), \quad \chi>0,
$$

which carries all the required information on the angle $\theta$.

In the corresponding quantum theory the eigenvalues of $\vec{K}$ are

$$
\vec{k}(\varphi)=k(\cos \varphi, \sin \varphi), \quad k>0,
$$

with $k^{2}$ the value of the Casimir operator $\vec{K}^{2}$; therefore $k$ is not "quantized." It also characterizes the irreducible unitary representations of $E(2)[19,37,38]$. We see below that the modulus $k$ can easily be integrated out, with only the essential pair $(\cos \theta, \sin \theta)$ left. 
Using series expansion for the exponentials we get

$$
\begin{gathered}
g(\vartheta, \vec{a}=0)=g(\vartheta)=e^{\vartheta \tilde{L}}=\left(\begin{array}{ccc}
\cos \vartheta & -\sin \vartheta & 0 \\
\sin \vartheta & \cos \vartheta & 0 \\
0 & 0 & 1
\end{array}\right), \\
g\left(\vartheta=0, a_{1}, a_{2}=0\right)=g\left(a_{1}\right)=e^{a_{1} \tilde{K}_{1}}=\left(\begin{array}{ccc}
1 & 0 & a_{1} \\
0 & 1 & 0 \\
0 & 0 & 1
\end{array}\right), \\
g\left(\vartheta=0, a_{1}=0, a_{2}\right)=g\left(a_{2}\right)=e^{a_{2} \tilde{K}_{2}}=\left(\begin{array}{ccc}
1 & 0 & 0 \\
0 & 1 & a_{2} \\
0 & 0 & 1
\end{array}\right) .
\end{gathered}
$$

The group element in relation (7) can then be written as

$$
g(\vartheta, \vec{a})=g\left(a_{2}\right) \circ g\left(a_{1}\right) \circ g(\vartheta) .
$$

We now arrive at a critical part of the paper: As in the case of the Heisenberg-Weyl group [24,25] the special group element [21,39]

$$
\begin{aligned}
& g_{0}(\vartheta, \vec{b})=\exp \left(b_{1} \tilde{K}_{1}+b_{2} \tilde{K}_{2}+\vartheta \tilde{L}\right)
\end{aligned}
$$

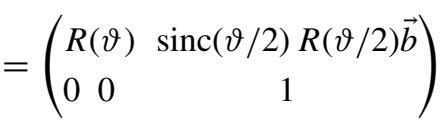

plays an essential role in the following construction of the Wigner function. Its importance has been emphasized previously by Wolf and collaborators [20-22].

Comparing Eq. (16) and Eq. (7) shows that the translations are now parametrized in an angle-dependent way. We have

$$
\vec{a}=\operatorname{sinc}(\vartheta / 2) R(\vartheta / 2) \vec{b} .
$$

Crucial for the rest of the paper is that the element $g_{0}$ from Eq. (16) can also be written as

$$
g_{0}(\vartheta, \vec{b})=e^{(\vartheta / 2) \tilde{L}} \circ e^{\operatorname{sinc}(\vartheta / 2) \vec{b} \cdot \overrightarrow{\tilde{K}}} \circ e^{(\vartheta / 2) \tilde{L}},
$$

which amounts to a kind of Weyl symmetrization. From Eq. (17) we then obtain

$$
g_{0}(\vartheta, \vec{a})=e^{(\vartheta / 2) \tilde{L}} \circ e^{R(-\vartheta / 2) \vec{a} \cdot \overrightarrow{\tilde{K}}} \circ e^{(\vartheta / 2) \tilde{L}} .
$$

Now the undesirable factor $\operatorname{sinc}(\vartheta / 2)$ has dropped out.

\section{B. Constructing the quantum mechanical Wigner operator}

In the quantum theory the group element, (19), is "promoted" to the unitary operator

$$
U_{0}(\vartheta, \vec{a})=e^{i(\vartheta / 2) L} \circ e^{i \vec{a}_{(-\vartheta / 2)} \cdot \vec{K}} \circ e^{i(\vartheta / 2) L} .
$$

[See Eq. (6) for notations.]

The unitary operator $U_{0}$ is supposed to act in an appropriate Hilbert space in which the Euclidean group is represented unitarily and in which the operators $L, K_{1}$, and $K_{2}$ are selfadjoint. Possible unitary representations are the irreducible, the quasiregular, and the regular ones [37]. We here consider only irreducible unitary representations of $E(2)$ [19,37-39].

For the construction of appropriate Wigner functions in the phase space, (3), we have to combine the operator, (20), in a suitable way with the classical variables $\vec{\chi}(\theta)$ [see Eq. (10)] and the angular momentum $p$. Following lessons from the Heisenberg-Weyl group [24,25] and from-appropriately modified-suggestions by Wolf et al. [20,21] the present proposal for a proper $(\theta, p)$ Wigner operator for the phase space with the topology $S^{1} \times \mathbb{R}$ is

$$
\begin{aligned}
V[\vec{\chi}(\theta), p] & =\frac{1}{(2 \pi)^{3}} \int_{-\pi}^{\pi} d \vartheta \int_{-\infty}^{\infty} d a_{1} d a_{2} \hat{U}_{0}, \\
\hat{U}_{0} & =e^{i(L-p)(\vartheta / 2)} \circ e^{i(\vec{K}-\vec{\chi}(\theta)) \cdot \vec{a}_{(-\vartheta / 2)}} \circ e^{i(L-p)(\vartheta / 2)} .
\end{aligned}
$$

This is a kind of ordered group averaging [with invariant Haar measure $d g(\vartheta, \vec{a})=d \vartheta d a_{1} d a_{2}$ ] of the differences between the classical quantities $\vec{\chi}(\theta)$ and $p$ and their corresponding operator counterparts. Ansatz (21) differs from that in Ref. [21] essentially by a different operator ordering, which avoids the obstructive explicit factor $\operatorname{sinc}(\vartheta / 2)$.

All irreducible unitary representations of $E(2)$ and itsinfinitely many-covering groups can be implemented in a Hilbert space $L^{2}\left(S^{1}, d \varphi / 2 \pi ; \delta\right)$ with the scalar product

$$
\left(\psi_{2}, \psi_{1}\right)=\int_{-\pi}^{\pi} \frac{d \varphi}{2 \pi} \psi_{2}^{*}(\varphi) \psi_{1}(\varphi)
$$

and a basis

$$
e_{n, \delta}(\varphi)=e^{i(n+\delta) \varphi},\left(e_{m, \delta}, e_{n, \delta}\right)=\delta_{m n}, m, n \in \mathbb{Z},
$$

where $\delta \in[0,1)$ characterizes the choice of a covering group [10,19]. A $\delta \neq 0$ becomes important for fractional orbital angular momenta [10]. $\delta_{m n}$ is, of course, the usual Kronecker symbol.

For any $\psi^{[\delta]}(\varphi) \in L^{2}\left(S^{1}, d \varphi / 2 \pi ; \delta\right)$, i.e., $\psi^{[\delta]}(\varphi+2 \pi)=$ $e^{i 2 \pi \delta} \psi^{[\delta]}(\varphi)$ we have the expansion

$$
\psi^{[\delta]}(\varphi)=\sum_{n \in \mathbb{Z}} c_{n} e_{n, \delta}(\varphi), c_{n}=\left(e_{n, \delta}, \psi^{[\delta]}\right) .
$$

The coefficients $c_{n}$ are independent of $\delta$. That dependence of $\psi^{[\delta]}(\varphi)$ is taken over by $e_{n, \delta}(\varphi)$.

In the following we put $\delta=0$. The general case $\delta \neq 0$ is briefly discussed in Sec. V A below.

The action of the self-adjoint operators $\vec{K}$ and $L$ is given by $(\hbar=1)$

$$
\begin{gathered}
\vec{K} \psi(\varphi)=k(\cos \varphi, \sin \varphi) \psi(\varphi), \quad L \psi(\varphi)=\frac{1}{i} \partial_{\varphi} \psi(\varphi), \\
e^{i \vec{a}_{(-\vartheta / 2)} \cdot \vec{K}} \psi(\varphi)=e^{i \vec{a}_{(-\vartheta / 2)} \cdot \vec{k}(\varphi)} \psi(\varphi), \\
e^{i \vartheta L} \psi(\varphi)=\psi(\varphi+\vartheta) .
\end{gathered}
$$

The functions $e_{n, \delta}(\varphi)$ obviously are eigenfunctions of $L$ with eigenvalues $n+\delta$, whereas $\vec{K}$ acts as a multiplication operator. Different values of $k$ belong to different irreducible representations and the Plancherel measure for the Fourier transforms on $E(2)$ is $k d k$ [37-39].

We here can see what the replacement of the proper classical phase space $S^{1} \times \mathbb{R}$ with $S^{1} \times \mathbb{Z}$ means (see also Ref. [10]): It amounts to putting $k=0$ in Eqs. (25) and (26), i.e., representing the translations of $E(2)$ - which correspond to the pair $(\cos \theta, \sin \theta)$ - by the identity (the translations form a normal subgroup), thus eliminating the observable "angle" altogether. What is left are infinitely many irreducible 
representations $\left\{e^{i n \varphi}, n \in \mathbb{Z}\right\}$ of $\operatorname{SO}(2)$ alone and the factor $\mathbb{Z}$ in $S^{1} \times \mathbb{Z}$ corresponds to that dual of $\operatorname{SO}(2)$. The angle $\theta$ then has to be introduced forcibly "by hand."

For the matrix elements

$$
V_{\psi_{2} \psi_{1}}^{(k)}[\vec{\chi}(\theta), p] \equiv\left(\psi_{2}, V[\vec{\chi}(\theta), p] \psi_{1}\right)
$$

we obtain from relations (26) and (27)

$$
\begin{aligned}
V_{\psi_{2} \psi_{1}}^{(k)}[\vec{\chi}(\theta), p]= & \frac{1}{(2 \pi)^{4}} \int d g(\vartheta, \vec{a}) d \varphi e^{-i \omega+\vec{k}(\varphi) \vec{a}_{(-\vartheta / 2)}} \\
& \times \psi_{2}^{*}(\varphi-\vartheta / 2) \psi_{1}(\varphi+\vartheta / 2) \\
\omega= & \vec{\chi}(\theta) \cdot \vec{a}_{(-\vartheta / 2)}+p \vartheta
\end{aligned}
$$

Introducing polar coordinates $\vec{a}=a(\cos \alpha, \sin \alpha)$ yields

$$
\begin{aligned}
V_{\psi_{2} \psi_{1}}^{(k)}= & \frac{1}{(2 \pi)^{4}} \int d \vartheta d \alpha a d a d \varphi e^{-i p \vartheta} \\
& \times e^{-i a \chi \cos (\theta-\alpha+\vartheta / 2)} e^{i a k \cos (\varphi-\alpha+\vartheta / 2)} \\
& \times \psi_{2}^{*}(\varphi-\vartheta / 2) \psi_{1}(\varphi+\vartheta / 2) .
\end{aligned}
$$

Because of the possible expansion, (24), it suffices to take $\psi_{2}(\varphi)=e_{m}(\varphi)$ and $\psi_{1}(\varphi)=e_{n}(\varphi)$

Recalling the integral representation

$$
J_{n}(x)=\frac{1}{2 \pi} \int_{-\pi}^{\pi} d \beta e^{i(x \sin \beta-n \beta)}, \quad n \in \mathbb{Z},
$$

of the Bessel functions [40] and their (Hankel transform) "completeness" relation [41]

$$
\int_{0}^{\infty} \text { da } a J_{m}(x a) J_{m}(y a)=\frac{1}{x} \delta(x-y),
$$

and using the orthonormality, (23), of the $e_{m}(\varphi)$ we finally get

$$
\begin{aligned}
V_{m n}^{(k)}(\theta, p)= & e^{i(n-m) \theta}\left(\frac{1}{k} \delta(k-\chi)\right) \frac{1}{(2 \pi)^{2}} \\
& \times \int_{-\pi}^{\pi} d \vartheta e^{i[(m+n) / 2-p] \vartheta} .
\end{aligned}
$$

\section{MAIN RESULTS}

\section{A. The phase-space Wigner-Moyal matrix}

Equation (33) shows explicitly that the scale factor $\chi$ is a redundant variable and can easily be eliminated: integration (averaging) with the Plancherel measure $k d k$ gives our surprisingly simple but powerful main result:

$$
\begin{aligned}
V_{m n}(\theta, p) & =\int_{0}^{\infty} d k k V_{m n}^{(k)}(\theta, p) \\
& =\frac{1}{(2 \pi)^{2}} e^{i(n-m) \theta} \int_{-\pi}^{\pi} d \vartheta e^{i[(n+m) / 2-p] \vartheta} \\
& =\frac{1}{2 \pi} e^{i(n-m) \theta} \operatorname{sinc} \pi[p-(m+n) / 2] .
\end{aligned}
$$

The infinite-dimensional Hermitian (Wigner-Moyal) matrix $V(\theta, p)=\left(V_{m n}(\theta, p)\right)$ has the properties

$$
\begin{gathered}
\int_{-\infty}^{\infty} d p V_{m n}(\theta, p)=\frac{1}{2 \pi} e^{i(n-m) \theta}, \\
\int_{-\pi}^{\pi} d \theta V_{m n}(\theta, p)=\operatorname{sinc} \pi(p-m) \delta_{m n}, \\
\int_{-\pi}^{\pi} d \theta \int_{-\infty}^{\infty} d p V_{m n}(\theta, p)=\delta_{m n}, \\
\operatorname{tr}\left(V_{m n}(\theta, p)\right)=\frac{1}{2 \pi} \sum_{n \in \mathbb{Z}} \operatorname{sinc} \pi(p-n)=\frac{1}{2 \pi}, \\
\int_{-\pi}^{\pi} d \theta \int_{-\infty}^{\infty} d p V_{k l}(\theta, p) V_{m n}(\theta, p)=\frac{1}{2 \pi} \delta_{k n} \delta_{l m}, \\
\left|V_{m n}\right| \leqslant \frac{1}{2 \pi},
\end{gathered}
$$

where the relations

$$
\begin{array}{r}
\operatorname{sinc} \pi x=1 \quad \text { for } \quad x=0, \\
|\operatorname{sinc} \pi x|<1 \quad \text { for } x \neq 0, \\
\int_{-\infty}^{\infty} d x \operatorname{sinc} \pi(x+a)=1, \quad a \in \mathbb{R}, \\
\frac{1}{2 \pi} \sum_{n \in \mathbb{Z}} e^{i n \beta}=\delta(\beta) \text { for } \beta \in[-\pi,+\pi]
\end{array}
$$

have been used. As for Eq. (39) see also Ref. [42].

It follows from Eq. (40) that

$$
\begin{aligned}
& \int_{-\pi}^{\pi} d \theta \int_{-\infty}^{\infty} d p V^{T}(\theta, p) \cdot V(\theta, p)=\frac{1}{2 \pi} \mathbf{1}, \\
& \int_{-\pi}^{\pi} d \theta \int_{-\infty}^{\infty} d p V(\theta, p) \cdot V^{T}(\theta, p)=\frac{1}{2 \pi} \mathbf{1},
\end{aligned}
$$

where $V^{T}\left(=V^{*}\right)$ is the transpose of matrix $V$ and $\mathbf{1}$ the unit operator in Hilbert space. Thus, $V$ is Hermitian and even orthogonal, but not unitary.

Another interesting property of $V(\theta, p)$ is

$$
\operatorname{tr}\left[V\left(\theta_{1}, p_{1}\right) \cdot V\left(\theta_{2}, p_{2}\right)\right]=\frac{1}{2 \pi} \delta\left(\theta_{1}-\theta_{2}\right) \operatorname{sinc} \pi\left(p_{1}-p_{2}\right) .
$$

Integrating this equation over the pair $\left(\theta_{1}, p_{1}\right)$ (or $\left.\left(\theta_{2}, p_{2}\right)\right)$ consistently yields relation (39). As mentioned in Sec. I the function sinc $\pi(p-m)$ interpolates the discrete quantum numbers $m$, etc., in terms of the continuous classical variable $p$ (for more details see the discussion in Sec. IV).

Note the following difference regarding integrations over angles above: whereas the integration measure in the scalar product, (22), is normalized as $d \phi /(2 \pi)$, the corresponding measure for the phase-space angle variable $\theta$ is $d \theta$.

For expansions

$$
\psi_{j}(\varphi)=\sum_{n \in \mathbb{Z}} c_{n}^{(j)} e_{n}(\varphi), \quad j=1,2 ; \quad c_{n}^{(j)}=\left(e_{n}, \psi_{j}\right),
$$


we get the "Moyal" function (see Refs. [4,24,25] for the corresponding $(q, p)$ case)

$$
\begin{aligned}
V_{\psi_{2} \psi_{1}}(\theta, p) & =\sum_{m, n \in \mathbb{Z}} c_{m}^{(2) *} V_{m n}(\theta, p) c_{n}^{(1)} \\
& =\frac{1}{(2 \pi)^{2}} \int_{-\pi}^{\pi} d \vartheta e^{-i p \vartheta} \psi_{2}^{*}(\theta-\vartheta / 2) \psi_{1}(\theta+\vartheta / 2) \\
& =\left(\psi_{2}, V(\theta, p) \psi_{1}\right),
\end{aligned}
$$

which, according to Eqs. (36)-(40), has the properties

$$
\begin{gathered}
\int_{-\infty}^{\infty} d p V_{\psi_{2} \psi_{1}}(\theta, p)=\frac{1}{2 \pi} \psi_{2}^{*}(\theta) \psi_{1}(\theta), \\
\int_{-\pi}^{\pi} d \theta V_{\psi_{2} \psi_{1}}(\theta, p)=\sum_{m \in \mathbb{Z}} c_{m}^{(2) *} \operatorname{sinc} \pi(p-m) c_{m}^{(1)} \\
\int_{-\infty}^{\infty} d p \int_{-\pi}^{\pi} d \theta V_{\psi_{2} \psi_{1}}(\theta, p)=\left(\psi_{2}, \psi_{1}\right) \\
\int_{-\infty}^{\infty} d p \int_{-\pi}^{\pi} d \theta V_{\psi_{2} \psi_{1}}^{*}(\theta, p) V_{\phi_{2} \phi_{1}}(\theta, p) \\
=\frac{1}{2 \pi}\left(\psi_{1}, \phi_{1}\right)\left(\psi_{2}, \phi_{2}\right)^{*}
\end{gathered}
$$

On the left-hand sides of the Eqs. (50)-(53) we have integrals over phase-space functions, whereas on the right-hand sides we have quantities from the corresponding Hilbert space, except for Eq. (51), where the function sinc $\pi(p-m)$ occurs. However, because of the orthonormality relation (references in Sec. IV)

$$
\int_{-\infty}^{\infty} d p \operatorname{sinc} \pi(m-p) \operatorname{sinc} \pi(n-p)=\delta_{m n},
$$

that sinc function can be eliminated immediately,

$$
\begin{aligned}
& \int_{-\infty}^{\infty} d p \operatorname{sinc} \pi(n-p) \int_{-\pi}^{\pi} d \theta V_{\psi_{2} \psi_{1}}(\theta, p) \\
& \quad=\int_{-\infty}^{\infty} d p \operatorname{sinc} \pi(n-p) \sum_{m \in \mathbb{Z}} c_{m}^{(2) *} \operatorname{sinc} \pi(p-m) c_{m}^{(1)} \\
& =c_{n}^{(2) *} c_{n}^{(1)}
\end{aligned}
$$

a relation which will become important in Sec. IV for $\psi_{2}=\psi_{1}$.

\section{B. Phase-space functions associated with Hilbert-space operators}

If $\psi_{1}(\varphi)=A \psi(\varphi)$, where $A$ is some operator (e.g., $\cos \varphi, \sin \varphi, L$, or functions of these), then Eq. (52) provides matrix elements of $A$, and for $\psi_{2}=\psi$ its expectation value $(\psi, A \psi)$, in terms of integrals over phase-space densities: For $\psi_{1}=A \psi$ we have

$$
c_{n}^{(1)}=\sum_{k \in \mathbb{Z}} c_{k}\left(e_{n}, A e_{k}\right)
$$

and it follows from Eq. (52) that

$$
\begin{aligned}
\left(\psi_{2}, A \psi\right) & =\int_{-\infty}^{\infty} d p \int_{-\pi}^{\pi} d \theta a_{\psi_{2} \psi}(\theta, p), \\
a_{\psi_{2} \psi}(\theta, p) & =\sum_{m, n \in \mathbb{Z}} \frac{1}{2} c_{m}^{(2) *}[V(\theta, p) \cdot A+A \cdot V(\theta, p)]_{m n} c_{n},
\end{aligned}
$$

which expresses a quantum mechanical matrix element in terms of a phase-space integral over an associated density $a_{\psi_{2} \psi}(\theta, p)$. The (Weyl) symmetrization of $V=\left(V_{m k}\right)$ and $A=\left(A_{k n}\right)$ in Eq. (57) is required for $a_{\psi \psi}(\theta, p)$ to be real if $A$ is Hermitian.

For $\psi_{2}=e_{m}, \psi=e_{n}$ we get

$$
\begin{aligned}
A_{m n} & \equiv\left(e_{m}, A e_{n}\right)=\int_{-\infty}^{\infty} d p \int_{-\pi}^{\pi} d \theta a_{m n}(\theta, p), \\
a_{m n}(\theta, p) & =\frac{1}{2}[V(\theta, p) \cdot A+A \cdot V(\theta, p)]_{m n},
\end{aligned}
$$

which can formally be written as

$$
A=\left(A_{m n}\right)=\int_{-\infty}^{\infty} d p \int_{-\pi}^{\pi} d \theta \frac{1}{2}[V(\theta, p) \cdot A+A \cdot V(\theta, p)] .
$$

From the diagonal elements

$$
a_{m}(\theta, p)=\frac{1}{2}[V(\theta, p) \cdot A+A \cdot V(\theta, p)]_{m m}
$$

we obtain

$$
\begin{aligned}
\operatorname{tr} A & =\sum_{m \in \mathbb{Z}} \int_{-\infty}^{\infty} d p \int_{-\pi}^{\pi} d \theta a_{m}(\theta, p) \\
& =\int_{-\infty}^{\infty} d p \int_{-\pi}^{\pi} d \theta \operatorname{tr}[A \cdot V(\theta, p)] .
\end{aligned}
$$

If $A$ is a diagonal density matrix,

$$
\rho=\left(\lambda_{n} \delta_{m n}\right), \quad \lambda_{n} \geqslant 0, \quad \sum_{n \in \mathbb{Z}} \lambda_{n}=1,
$$

then we have

$$
\begin{aligned}
& \operatorname{tr}[\rho \cdot V(\theta, p)]=\frac{1}{2 \pi} \sum_{m \in \mathbb{Z}} \lambda_{m} \operatorname{sinc} \pi(p-m), \\
& \int_{-\infty}^{\infty} d p \int_{-\pi}^{\pi} d \theta \operatorname{tr}[\rho \cdot V(\theta, p)]=\sum_{m \in \mathbb{Z}} \lambda_{m}=1 .
\end{aligned}
$$

Using relations (54) the probabilities $\lambda_{m}$ can be extracted from Eq. (63):

$$
\lambda_{m}=2 \pi \int_{-\infty}^{\infty} d p \operatorname{sinc} \pi(p-m) \operatorname{tr}[\rho \cdot V(\theta, p)] .
$$

As $A$ in Eq. (61) is any (trace class) operator we may take $A=O \cdot \rho$, where $\rho$ is a density matrix and $O$ a self-adjoint observable. We then have

$$
\operatorname{tr}(\rho \cdot O)=\int_{-\infty}^{\infty} d p \int_{-\pi}^{\pi} d \theta \frac{1}{2} \operatorname{tr}[V(\theta, p) \cdot\{O, \rho\}] .
$$

As $\operatorname{tr}(\rho \cdot O)=\operatorname{tr}(O \cdot \rho)$ the right-hand side of Eq. (66) has to be symmetrized in $\rho$ and $O$ too.

The phase-space representation of the trace $\operatorname{tr}(A \cdot B)$ of a product $A \cdot B$ can also be dealt with in a very similar way 
as in the $(q, p)$ case $[3-5,7,43]$. Let us write $\operatorname{tr}[A \cdot V(\theta, p)]$ explicitly,

$$
\begin{aligned}
\operatorname{tr} & {[A \cdot V(\theta, p)] } \\
& =\sum_{m, n \in \mathbb{Z}} A_{m n} V_{n m}(\theta, p) \\
& =\frac{1}{(2 \pi)^{2}} \sum_{m, n \in \mathbb{Z}} A_{m n} e^{i(m-n) \theta} \int_{-\pi}^{\pi} d \vartheta e^{i[(m+n) / 2-p] \vartheta},
\end{aligned}
$$

with the corresponding expression for $\operatorname{tr}[B \cdot V(\theta, p)]$. Inserting these into

$$
\int_{-\infty}^{\infty} d p \int_{-\pi}^{\pi} d \theta \operatorname{tr}[A \cdot V(\theta, p)] \operatorname{tr}[B \cdot V(\theta, p)]
$$

and carrying out the integrations yield the important relation $\operatorname{tr}(A \cdot B)=2 \pi \int_{-\infty}^{\infty} d p \int_{-\pi}^{\pi} d \theta \operatorname{tr}[A \cdot V(\theta, p)] \operatorname{tr}[B \cdot V(\theta, p)]$.

Especially, we have, for the expectation value of the operator $O$ for a given $\rho$,

$$
\begin{aligned}
\langle O\rangle_{\rho} & =\operatorname{tr}(\rho \cdot O) \\
& =2 \pi \int_{-\infty}^{\infty} d p \int_{-\pi}^{\pi} d \theta \operatorname{tr}[\rho \cdot V(\theta, p)] \operatorname{tr}[O \cdot V(\theta, p)],
\end{aligned}
$$

with $\operatorname{tr}[\rho \cdot V(\theta, p)]$ from Eq. (63).

\section{Time evolution}

Inserting expansions (48) into their Schrödinger equations

$$
i \partial_{t} \psi_{j}(t, \varphi)=H \psi_{j}(t, \varphi)
$$

implies

$$
i \dot{c}_{m}^{(j)}(t)=\sum_{n \in \mathbb{Z}} c_{n}^{(j)}(t)\left(e_{m}, H e_{n}\right)
$$

which leads to the Schrödinger time evolution

$$
\partial_{t} V_{\psi_{2} \psi_{1}}(\theta, p ; t)=i\left(\psi_{2}(t),[H, V] \psi_{1}(t)\right) .
$$

That is, the time evolution of $V_{\psi_{2} \psi_{1}}$ is determined by the operator (matrix)

$$
K(\theta, p)=i[H, V(\theta, p)],
$$

which is Hermitian if $H$ and $V$ are Hermitian and for which $\operatorname{tr} K=0$.

A simple example is

$$
H=\varepsilon L^{2}, \quad\left(e_{m}, H e_{n}\right)=\varepsilon n^{2} \delta_{m n},
$$

which implies the matrix

$$
\left(K_{m n}(\theta, p)\right), K_{m n}=\left(e_{m}, K e_{n}\right)=i \varepsilon\left(m^{2}-n^{2}\right) V_{m n}(\theta, p) .
$$

Evolution (73) holds, of course, also for the Wigner function proper $V_{\psi}(\theta, p ; t)$, for which $\psi_{2}=\psi_{1}=\psi$ in Eq. (49) and which is discussed in the next section. by

There it is also discussed that the Wigner function is given

$$
V_{\rho}(\theta, p ; t)=\operatorname{tr}[\rho(t) \cdot V(\theta, p)]
$$

if a state is characterized not by a single wave function $\psi(t)$ but by a density matrix $\rho(t)$, which obeys the von Neumann equation (we are still in the Schrödinger picture):

$$
\partial_{t} \rho(t)=-i[H, \rho(t)] \text {. }
$$

Inserting this into

$$
\partial_{t} V_{\rho}(\theta, p ; t)=\operatorname{tr}\left[\partial_{t} \rho(t) \cdot V(\theta, p)\right]
$$

yields

$$
\partial_{t} V_{\rho}(\theta, p ; t)=\operatorname{tr}[\rho \cdot K(\theta, p)],
$$

where $K(\theta, p)$ is defined in Eq. (74).

\section{WIGNER FUNCTION FOR A GIVEN STATE}

\section{A. General properties}

If $\psi_{2}=\psi_{1}=\psi$ in Eq. (49), then the real function

$$
\begin{aligned}
V_{\psi}(\theta, p) & =\frac{1}{(2 \pi)^{2}} \int_{-\pi}^{\pi} d \vartheta e^{-i p \vartheta} \psi^{*}(\theta-\vartheta / 2) \psi(\theta+\vartheta / 2) \\
& =\sum_{m, n \in \mathbb{Z}} c_{m}^{*} V_{m n}(\theta, p) c_{n} \\
& =(\psi, V(\theta, p) \psi)
\end{aligned}
$$

is the strict analog of the original Wigner function. According to Eqs. (50)-(53) and (55) it obeys

$$
\int_{-\infty}^{\infty} d p V_{\psi}(\theta, p)=\frac{1}{2 \pi}|\psi(\theta)|^{2}
$$

$$
\begin{gathered}
\int_{-\pi}^{\pi} d \theta V_{\psi}(\theta, p)=\sum_{n \in \mathbb{Z}}\left|c_{n}\right|^{2} \operatorname{sinc} \pi(p-n) \equiv \omega_{\psi}(p), \\
\int_{-\infty}^{\infty} d p \omega_{\psi}(p) \operatorname{sinc} \pi(p-m)=\left|c_{m}\right|^{2}, \\
\int_{-\infty}^{\infty} d p \int_{-\pi}^{\pi} d \theta V_{\psi}(\theta, p)=\sum_{n \in \mathbb{Z}}\left|c_{n}\right|^{2}=1, \\
\int_{-\infty}^{\infty} d p \int_{-\pi}^{\pi} d \theta V_{\psi_{2}}(\theta, p) V_{\psi_{1}}(\theta, p)=\frac{1}{2 \pi}\left|\left(\psi_{2}, \psi_{1}\right)\right|^{2} .
\end{gathered}
$$

In addition, the inequality

$$
\left|V_{\psi}(\theta, p)\right| \leqslant 1 / \pi
$$

holds. It follows from Schwarz's inequality as follows: Expression (81) can be written as

$$
\begin{aligned}
V_{\psi}(\theta, p) & =\frac{1}{2 \pi}\left(\chi_{2}, \chi_{1}\right), \\
\chi_{1}(\theta, p ; \vartheta) & =e^{-i p \vartheta / 2} \psi(\theta+\vartheta / 2), \\
\chi_{2}(\theta, p ; \vartheta) & =e^{i p \vartheta / 2} \psi(\theta-\vartheta / 2) .
\end{aligned}
$$

Because

$$
\left|\left(\chi_{2}, \chi_{1}\right)\right|^{2} \leqslant\left(\chi_{2}, \chi_{2}\right)\left(\chi_{1}, \chi_{1}\right)
$$


and

$$
\begin{aligned}
\left(\chi_{1}, \chi_{1}\right) & =\int_{-\pi}^{\pi} \frac{d \vartheta}{2 \pi}|\psi(\vartheta / 2+\theta)|^{2} \\
& =2 \int_{-\pi / 2}^{\pi / 2} \frac{d \beta}{2 \pi}|\psi(\beta)|^{2} \\
& \leqslant 2(\psi, \psi)=2,
\end{aligned}
$$

with the same for $\left(\chi_{2}, \chi_{2}\right)$, inequality (87) follows.

Equation (81) gives the Wigner function for a pure state $\psi$. It can immediately be generalized to a mixed state characterized by a density matrix $\rho$. First, we rewrite the scalar product, (81), as the trace of two operators: If $P_{\psi}$ is the projection operator onto state $\psi\left(P_{\psi}=|\psi\rangle\langle\psi|\right.$ in Dirac's notation $)$, then its matrix elements are given by

$$
\left(P_{\psi}\right)_{m n}=c_{m} c_{n}^{*} .
$$

Therefore Eq. (81) can be written as

$$
V_{\psi}(\theta, p)=(\psi, V(\theta, p) \psi)=\operatorname{tr}\left[P_{\psi} \cdot V(\theta, p)\right] .
$$

As $P_{\psi}$ is a special density matrix the generalization of $V_{\psi}$ to a mixed state with density matrix $\rho$ is obviously

$$
V_{\rho}(\theta, p)=\operatorname{tr}[\rho \cdot V(\theta, p)],
$$

a quantity we already know from the preceding section.

Again using relations (36)-(40) we now have, instead of Eqs. (82)-(86),

$$
\begin{gathered}
\int_{-\infty}^{\infty} d p V_{\rho}(\theta, p)=\frac{1}{2 \pi} \sum_{m, n \in \mathbb{Z}} e_{m}(\theta) \rho_{m n} e_{n}^{*}(\theta), \\
\int_{-\pi}^{\pi} d \theta V_{\rho}(\theta, p)=\sum_{m \in \mathbb{Z}} \rho_{m m} \operatorname{sinc} \pi(p-m) \equiv \omega_{\rho}(p), \\
\int_{-\infty}^{\infty} d p \omega_{\rho}(p) \operatorname{sinc} \pi(p-m)=\rho_{m m} \\
\int_{-\infty}^{\infty} d p \int_{-\pi}^{\pi} d \theta V_{\rho}(\theta, p)=\sum_{m \in \mathbb{Z}} \rho_{m m}=\operatorname{tr}(\rho)=1 \\
\int_{-\infty}^{\infty} d p \int_{-\pi}^{\pi} d \theta V_{\rho_{2}}(\theta, p) V_{\rho_{1}}(\theta, p)=\frac{1}{2 \pi} \operatorname{tr}\left(\rho_{2} \cdot \rho_{1}\right)
\end{gathered}
$$

Equation (96) gives only the diagonal elements of the density matrix $\rho$. Its generalization follows immediately from relation (40),

$$
\int_{-\infty}^{\infty} d p \int_{-\pi}^{\pi} d \theta V_{k l}(\theta, p) V_{\rho}(\theta, p)=\frac{1}{2 \pi} \rho_{k l},
$$

of which Eq. (96) is a special case.

The representations of the Wigner functions, (92) and (93), as traces make their invariance under unitary transformations explicit, especially under those of Eqs. (26) and (27).

\section{B. Marginal densities}

For the Wigner function $W_{\psi}(q, p)$ in the classical phase space $\left\{(q, p) \in \mathbb{R}^{2}\right\}$ one has the marginal quantum mechanical distributions

$$
\begin{aligned}
& \int d p W_{\psi}(q, p)=|\psi(q)|^{2}, \\
& \int d q W_{\psi}(q, p)=|\hat{\psi}(p)|^{2}
\end{aligned}
$$

for $q$ and $p$ separately. Properties (100) and (101) constitute one of the main requirements the Wigner function $W_{\psi}(q, p)$ should fulfill $[2-5,7,24,25]$.

At first glance these properties do hold in our case only for the marginal density $|\psi(\theta)|^{2}$ in Eq. (82). However, the situation here is only slightly more complicated but, in principle, as straightforward as in the $(q, p)$ case: The quantum mechanical marginal probabilities for the quantized angular momentum are the discontinuous numbers

$$
b_{m}=\left|c_{m}\right|^{2}, \quad m \in \mathbb{Z},
$$

whereas the $\theta$ integration in Eq. (83) yields "only" the function $\omega_{\psi}(p)$. But Eq. (84) shows how the marginal probabilities, (102), can be extracted uniquely from $\omega_{\psi}(p)$.

The density $\omega_{\psi}(p)$ of Eq. (83) is an example of Whittaker's famous cardinal function $C(f, h)(x)$ associated with a function $f(x)$ the values of which are known only for a discrete subset of the otherwise continuous arguments $x$ [26], an important case well known from interpolation, sampling, and signal processing theories (see the reviews [27-34]),

$$
C(f, h)(x)=\sum_{n \in \mathbb{Z}} f(n h) \operatorname{sinc}[\pi(x-n h) / h],
$$

where $h$ is the step size.

In our case we have

$$
\begin{aligned}
C(g, h=1)(p) & =\sum_{m \in \mathbb{Z}}\left[g(p=m)=b_{m}\right] \operatorname{sinc} \pi(m-p) \\
& =\omega_{\psi}(p)
\end{aligned}
$$

for a possible function $g(p), p \in \mathbb{R}$, which now interpolates the different discrete values $\left|c_{m}\right|^{2}$, i.e., $g(p=m)=\left|c_{m}\right|^{2}$. The interval $h$ between adjacent supporting points here has the value $h=1$. If the Fourier transform $\hat{f}(u)$ of $f(x)$ is "band limited," i.e., $\hat{f}(u)$ vanishes outside the interval $u \in[-\pi / h, \pi / h]$ (Palais-Wiener case), then one even has $C(f, h)(x)=f(x)$ (Whittaker-Shannon sampling theorem, mathematically proved by Hardy [44]).

The functions $\operatorname{sinc} \pi(p-m), m \in \mathbb{Z}$, of Eq. (54) form a complete orthonormal basis for the Hilbert space of such functions $g(p)$ on the real line $\mathbb{R}$ (see also Ref. [45]).

Here then is the complete set of marginal densities from above [Eqs. (82), (84), (94), and (99)] for a wave function $\psi(\varphi)=\sum_{n \in \mathbb{Z}} c_{n} e_{n}(\varphi)$ or a density matrix $\rho=\left(\rho_{m n}\right)$ :

$$
\begin{gathered}
\frac{1}{2 \pi}|\psi(\theta)|^{2}=\int_{-\infty}^{\infty} d p V_{\psi}(\theta, p), \\
\left|c_{n}\right|^{2}=\int_{-\infty}^{\infty} d p \int_{-\pi}^{\pi} d \theta \operatorname{sinc} \pi(p-n) V_{\psi}(\theta, p), \\
\frac{1}{2 \pi} \sum_{m, n \in \mathbb{Z}} \rho_{m n} e_{m}(\theta) e_{n}^{*}(\theta)=\int_{-\infty}^{\infty} d p V_{\rho}(\theta, p), \\
\frac{1}{2 \pi} \rho_{m n}=\int_{-\infty}^{\infty} d p \int_{-\pi}^{\pi} d \theta V_{m n}(\theta, p) V_{\rho}(\theta, p) .
\end{gathered}
$$




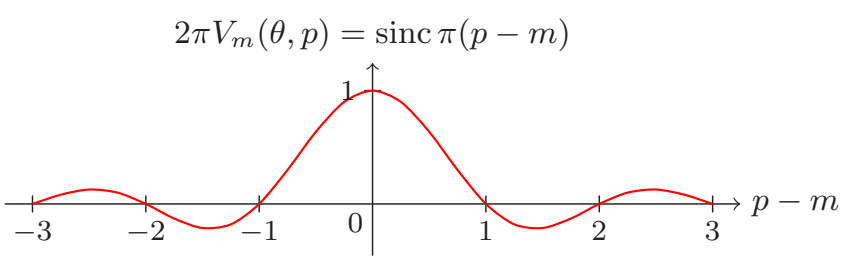

FIG. 1. The Wigner function $V_{m}(\theta, p)$, Eq. (109), for the basis function. $e_{m}(\varphi)$.

\section{Examples}

\section{Wigner function of the basis function $e_{m}(\varphi)$}

If $c_{m}=1$ and $c_{n}=0$ for $n \neq m$, then the diagonal matrix element

$$
V_{m}(\theta, p)=(1 / 2 \pi) \operatorname{sinc} \pi(p-m)
$$

is the Wigner function of the basis function $e_{m}(\varphi)$. The corresponding graph is shown in Fig. 1.

The Wigner function, (109), is independent of $\theta$ and can become negative as a function of $p$, e.g., in the interval $\pm(p-m) \in(1,2)$. So a Wigner function on $S^{1} \times \mathbb{R}$ can have negative values too, as in the $(q, p)$ case: The Wigner function (phase-space density) $W_{n}(q, p)$ for the $n$th energy level of the quantized harmonic oscillator has the value $\lambda(-1)^{n}, \lambda>0$ at $(q=0, p=0)$ [46]. In the $(q, p)$ case negativity of Wigner functions on certain subsets of the classical phase space is interpreted as a consequence of quantum effects. The same interpretation applies to the Wigner function here: Whereas the classical angular momentum $p$ is continuous, its quantized counterpart is discrete. This is reflected by the properties of the Wigner function, (109). The difference between the $(q, p)$ case and the $(\theta, p)$ case is that in the former the basic variables $q$ and $p$ in general remain continuous in the quantum theory too, whereas in the latter the basic angular momentum variable $p$ becomes discontinuous.

Integrals (82)-(85) become trivial here:

$$
\begin{gathered}
\int_{-\infty}^{\infty} d p V_{m}(\theta, p)=\frac{1}{2 \pi}, \\
\int_{-\pi}^{\pi} d \theta V_{m}(\theta, p)=\operatorname{sinc} \pi(p-m)=\omega_{m}(p), \\
\int_{-\infty}^{\infty} d p \omega_{m}(p) \operatorname{sinc} \pi(p-m)=\left|c_{m}\right|^{2}=1, \\
\int_{-\infty}^{\infty} d p \int_{-\pi}^{\pi} d \theta V_{m}(\theta, p)=1 .
\end{gathered}
$$

\section{Wigner functions of simple cat states}

The eigenstates $e_{+1}(\varphi)$ and $e_{-1}(\varphi)$ of the angular momentum operator $L$ with eigenvalues $m= \pm 1$ are the first excited states of a rotator with the simple Hamiltonian $H=\varepsilon L^{2}$, both with the same energy eigenvalue $\varepsilon$. They represent, however, two different types of rotations: clockwise and counterclockwise. The Wigner functions of their simple superpositions (cat states),

$$
\begin{aligned}
f_{+1,-1:+}(\varphi) & =\frac{1}{\sqrt{2}}\left[e_{m=1}(\varphi)+e_{m=-1}(\varphi)\right] \\
& =\frac{1}{\sqrt{2}}\left[e^{i \varphi}+e^{-i \varphi}\right]=\sqrt{2} \cos \varphi, \\
\left|f_{+1,-1:+}(\varphi)\right|^{2} & =1+\cos 2 \varphi=2 \cos ^{2} \varphi, \\
f_{+1,-1:-}(\varphi) & =\frac{1}{\sqrt{2}}\left[e_{m=1}(\varphi)-e_{m=-1}(\varphi)\right] \\
& =\frac{1}{\sqrt{2}}\left[e^{i \varphi}-e^{-i \varphi}\right]=i \sqrt{2} \sin \varphi, \\
\left|f_{+1,-1:-}(\varphi)\right|^{2} & =1-\cos 2 \varphi,
\end{aligned}
$$

show in an interesting manner the influence of the interference or entanglement term $\cos 2 \varphi$ for the corresponding phasespace densities $V_{f_{ \pm}}(\theta, p)$ [in the following we write $f_{+}(\varphi)$ for $\left.f_{+1,-1:+}(\varphi)\right]$.

Inserting $f_{+}(\varphi)$ into Eq. (81) we get its Wigner function

$$
\begin{aligned}
2 \pi V_{f_{+}}(\theta, p)= & \cos 2 \theta \operatorname{sinc} \pi p \\
& +\frac{1}{2}[\operatorname{sinc} \pi(p+1)+\operatorname{sinc} \pi(p-1)] .
\end{aligned}
$$

The integrals (82)-(85) now take the form

$$
\begin{gathered}
\int_{-\infty}^{\infty} d p V_{f_{+}}(\theta, p)=\frac{1}{2 \pi}(\cos 2 \theta+1) \\
\int_{-\pi}^{\pi} d \theta V_{f_{+}}(\theta, p)=\frac{1}{2}[\operatorname{sinc} \pi(p+1)+\operatorname{sinc} \pi(p-1)] \\
\equiv \omega_{f_{+}}(p) \\
\int_{-\infty}^{\infty} d p \omega_{f_{+}}(p) \operatorname{sinc} \pi(p+1)=\left|c_{-1}\right|^{2}=\frac{1}{2} \\
\int_{-\infty}^{\infty} d p \omega_{f_{+}}(p) \operatorname{sinc} \pi(p-1)=\left|c_{+1}\right|^{2}=\frac{1}{2} \\
\int_{-\infty}^{\infty} d p \int_{-\pi}^{\pi} d \theta V_{f_{+}}(\theta, p)=1
\end{gathered}
$$

The $\theta$-dependent term in Eq. (118) represents the interference or entanglement part of the probability density, (115). Graphs of function (118)-shown in Fig. 2-parametrized by different angles $\theta \in[-\pi,+\pi]$ demonstrate the strong influence that the interference term has on the phase-space function $V_{f_{+}}(\theta, p)$.

The Wigner function $V_{f_{-}}$for state (116) is

$$
\begin{aligned}
2 \pi V_{f_{-}}(\theta, p)= & -\cos 2 \theta \operatorname{sinc} \pi p \\
& +\frac{1}{2}[\operatorname{sinc} \pi(p+1)+\operatorname{sinc} \pi(p-1)]
\end{aligned}
$$

and can be discussed in the same way as that for $f_{+}$.

Both $f_{+}(\varphi)$ and $f_{-}(\varphi)$ are special cases of $f_{\alpha}(\varphi)=$ $1 / \sqrt{2}\left(e^{i \varphi}+e^{-i \alpha} e^{-i \varphi}\right)$, which leads to the same Wigner function as in Eq. (118), with $2 \theta$ replaced by $2 \theta+\alpha$.

\section{Minimal uncertainty states}

The following example of a special state is taken from Sec. III in Ref. [10] (for a related later discussion see Ref. [47]): 


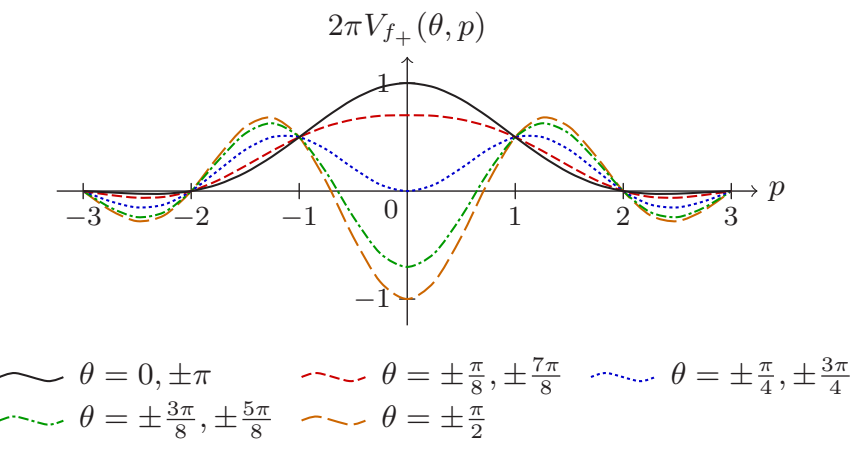

FIG. 2. Graphs of the Wigner function $2 \pi V_{f_{+}}(\theta, p)=$ $\cos 2 \theta \operatorname{sinc} \pi p+\frac{1}{2}[\operatorname{sinc} \pi(p+1)+\operatorname{sinc} \pi(p-1)]$ for the cat state $f_{+}(\varphi)=\frac{1}{\sqrt{2}}\left[e_{m=1}(\varphi)+e_{m=-1}(\varphi)\right]$ as a continuous function of the classical angular momentum $p$ for different discrete values of the phase-space angle $\theta \in[-\pi,+\pi]$. Note the special cases $2 \pi V_{f_{+}}(\theta, p=0)=\cos 2 \theta$ and $2 \pi V_{f_{+}}(\theta, p= \pm 1)=1 / 2$.

If $A$ and $B$ are two (noncommuting) self-adjoint operators and if state $\psi$ belongs to their common domain of definition, then the general "uncertainty relation" [48-50]

$$
(\Delta A)_{\psi}^{2}(\Delta B)_{\psi}^{2} \geqslant\left|\left\langle S_{\psi}(A, B)\right\rangle_{\psi}\right|^{2}+\frac{1}{4}\left|\langle[A, B]\rangle_{\psi}\right|^{2}
$$

holds, where

$$
\begin{gathered}
(\Delta A)_{\psi}^{2}=\left\langle\left(A-\langle A\rangle_{\psi}\right)^{2}\right\rangle_{\psi}, \quad\langle A\rangle_{\psi}=(\psi, A \psi), \\
S_{\psi}(A, B)=\frac{1}{2}(A B+B A)-\langle A\rangle_{\psi}\langle B\rangle_{\psi} .
\end{gathered}
$$

Of special interest are those states $\psi=\psi_{e}$ for which inequality (125) becomes an equality. That equality holds iff $\psi_{e}$ obeys the linear dependence equation

$$
\left(B-\langle B\rangle_{\psi_{e}}\right) \psi_{e}=\sigma\left(A-\langle A\rangle_{\psi_{e}}\right) \psi_{e}, \quad \sigma=\gamma+i s,
$$

where the given real parameters $\gamma$ and $s$ determine the following statistical quantities:

$$
\begin{gathered}
\frac{\left\langle S_{\psi_{e}}(A, B)\right\rangle_{\psi_{e}}}{(\Delta A)_{\psi_{e}}^{2}}=\gamma, \\
\frac{1}{2 i} \frac{\langle[A, B]\rangle_{\psi_{e}}}{(\Delta A)_{\psi_{e}}^{2}}=s, \\
\frac{(\Delta B)_{\psi_{e}}^{2}}{(\Delta A)_{\psi_{e}}^{2}}=|\sigma|=\sqrt{\gamma^{2}+s^{2}} .
\end{gathered}
$$

In the usual case $A=Q, B=P$ the solutions $\psi_{e}$ of Eq. (128) are Gaussian wave packets (coherent states), for which $\gamma=0$ :

$$
\begin{aligned}
\psi_{e}(x) & =(s / \pi)^{1 / 4} e^{-(s / 2)\left(x-x_{e}\right)^{2}} e^{i p_{e} x}, \quad s>0, \\
x_{e} & =\langle Q\rangle_{\psi_{e}}, \quad p_{e}=\langle P\rangle_{\psi_{e}} .
\end{aligned}
$$

In our case we take $A=S=\sin \varphi,\langle S\rangle_{\psi_{e}}=0$, and $B=L=$ $(1 / i) \partial_{\varphi}$ (in Ref. [10] Sec. III starts with $A=C=\cos \varphi$, but $A=\sin \varphi$ is closer to the standard von Mises statistical distribution [51,52]). Assuming here that $\gamma=0$ too, the choice $A=\sin \varphi$ and $B=L$ yields the following normalized solution of Eq. (128):

$$
\begin{gathered}
\psi_{e}(\varphi)=\frac{1}{\sqrt{I_{0}(2 s)}} e^{i p_{e} \varphi+s \cos \varphi}, \quad s>0, \\
\left|\psi_{e}(\varphi)\right|^{2}=\frac{e^{2 s \cos \varphi}}{I_{0}(2 s)}, \\
p_{e}=\langle L\rangle_{\psi_{e}}, \\
\langle S\rangle_{\psi_{e}}=0 \\
\langle C\rangle_{\psi_{e}}=\frac{I_{1}(2 s)}{I_{0}(2 s)} .
\end{gathered}
$$

If one allowed for $S_{e}=\langle S\rangle_{\psi_{e}} \neq 0$ in Eq. (128), solution (133) would include a nonperiodic factor $\exp \left(s S_{e} \varphi\right)$, which cannot be incorporated into any Hilbert space with a scalar product, (22), and a basis, (23). Similarly, solution (132) of Eq. (128) is not square-integrable on the real line for $s<0$.

The functions $I_{n}(x)$ are modified Bessel functions (for more details which we do not need here see Ref. [10]). The following integral representation for $n \in \mathbb{Z}$ has been and will be used [53]:

$$
I_{n}(z)=\frac{1}{2 \pi} \int_{-\pi}^{\pi} d \phi e^{z \cos \phi+i n \phi}=I_{-n}(z) .
$$

$I_{n}(z)$ is real for real $z$ and $I_{n}(-z)=(-1)^{n} I_{n}(z)$. For the shapes of distribution (134) for different values of $s$ see Fig. 3.1 in Ref. [52].

As

$$
\psi_{e}(\varphi+2 \pi)=e^{i 2 \pi p_{e}} \psi_{e}(\varphi),
$$

function (133) is generally not periodic, because $p_{e}$ can be any real number. However, its treatment can be reduced to the case discussed in the context of Eqs. (23) and (24): We decompose the real number $p_{e}$ uniquely into an integer $n_{e}$ and a fractional rest,

$$
p_{e}=n_{e}+\delta, \delta \in[0,1),
$$

so that

$$
\psi_{e}(\varphi)=\frac{1}{\sqrt{I_{0}(2 s)}} e^{i\left(n_{e}+\delta\right) \varphi+s \cos \varphi} .
$$

This yields the expansion coefficients

$$
\begin{aligned}
c_{m} & =\left(e_{m, \delta}, \psi_{e}\right) \\
& =\frac{1}{\sqrt{I_{0}(2 s)}} \int_{-\pi}^{\pi} \frac{d \varphi}{2 \pi} e^{-i\left(m-n_{e}\right) \varphi+s \cos \varphi} \\
& =\frac{1}{\sqrt{I_{0}(2 s)}} I_{m-n_{e}}(s) .
\end{aligned}
$$

In order to calculate the Wigner function of state (133) we have to use the Wigner-Moyal matrix, (172), in the next section: We then get

$$
V_{\psi_{e}}^{[\delta]}(\theta, p)=\sum_{m . n \in \mathbb{Z}} c_{m}^{*} V_{m n}^{[\delta]}(\theta, p) c_{n} .
$$

Using for $c_{m}, c_{n}$, and $V_{m n}^{[\delta]}(\theta, p)$ the integral representations (142) and (172) below and observing relation (44) (twice) 


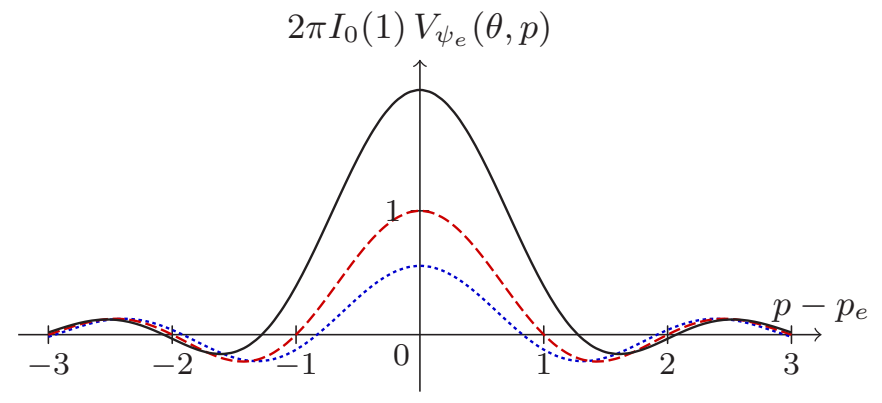

$$
\simeq \theta=0 \quad \cdots, \theta= \pm \pi / 2 \quad \cdots \cdots \cdots \cdot \theta= \pm \pi
$$

FIG. 3. Graphs of the Wigner function, (144), for state (133) with $s=1 / 2$. The curves show $2 \pi I_{0}(1) V_{\psi_{e}}(\theta, p)$ for $\theta=0, \pm \pi / 2, \pm \pi$ as a function of $p-p_{e}$. Numerically, $I_{0}(1)=1.2661$ [54].

yields the integral representation

$$
\begin{aligned}
& V_{\psi_{e}}^{[\delta]}(\theta, p) \\
& \quad=\frac{1}{(2 \pi)^{2} I_{0}(2 s)} \int_{-\pi}^{\pi} d \vartheta e^{-i\left(p-p_{e}\right) \vartheta+2 s \cos \theta \cos (\vartheta / 2)} \\
& \quad=\frac{1}{2 \pi^{2} I_{0}(2 s)} \int_{0}^{\pi} d \vartheta \cos \left[\left(p-p_{e}\right) \vartheta\right] e^{2 s \cos \theta \cos (\vartheta / 2)} \\
& p_{e}=n_{e}+\delta .
\end{aligned}
$$

We drop the label $[\delta]$ of $V_{\psi_{e}}^{[\delta]}(\theta, p)$ in the following.

For values $\theta= \pm \pi / 2$ we have

$$
V_{\psi_{e}}(\theta= \pm \pi / 2, p)=\frac{1}{2 \pi I_{0}(2 s)} \operatorname{sinc} \pi\left(p-p_{e}\right)
$$

The variables $p$ and $p_{e}$ are to be treated independently ( $p_{e}$ is the given"observable" $\langle L\rangle_{\psi_{e}}, p$ a phase-space variable). The curves in Fig. 3 have their main maximum for $p=p_{e}$.

The Wigner function, (144), indeed obeys the general relations (82)-(85) explicitly: Integrating Eq. (144) over $p$ gives a delta function $\delta(\vartheta)$ which makes the $\vartheta$ integral trivial. The result is the expected marginal density, (134):

$$
\int_{-\infty}^{\infty} d p V_{\psi_{e}}(\theta, p)=\frac{1}{2 \pi I_{0}(2 s)} e^{2 s \cos \theta}=\frac{1}{2 \pi}\left|\psi_{e}(\theta)\right|^{2} .
$$

The $\theta$ integration and the ensuing determination of $\left|c_{m}\right|^{2}$ are slightly more subtle,

$$
\begin{aligned}
& \int_{-\pi}^{\pi} d \theta V_{\psi_{e}}(\theta, p) \\
& \quad=\omega_{\psi_{e}}(p) \\
& =\frac{1}{2 \pi I_{0}(2 s)} \int_{-\pi}^{\pi} d \vartheta e^{-i\left(p-p_{e}\right) \vartheta} I_{0}[2 s \cos (\vartheta / 2)] \\
& =\frac{1}{\pi I_{0}(2 s)} \int_{0}^{\pi} d \vartheta \cos \left[\left(p-p_{e}\right) \vartheta\right] I_{0}[2 s \cos (\vartheta / 2)],
\end{aligned}
$$

where again relation (138) has been used.

In order to extract from $\omega_{\psi_{e}}$ the marginal probabilities $\left|c_{m}\right|^{2}$-according to Eq. (84) - we have to multiply $\omega_{\psi_{e}}(p)$ with sinc $\pi(p-m-\delta)$ and integrate over $p$ :

$$
\int_{-\infty}^{\infty} d p \omega_{\psi_{e}}(p) \operatorname{sinc} \pi(p-m-\delta) .
$$

Inserting

$$
\operatorname{sinc} \pi(p-m-\delta)=\frac{1}{2 \pi} \int_{-\pi}^{\pi} d \alpha e^{i(p-m-\delta) \alpha}
$$

into Eq. (148) yields a delta function $\delta(\alpha-\vartheta)$ and

$$
\begin{aligned}
& \frac{1}{2 \pi I_{0}(2 s)} \int_{-\pi}^{\pi} d \vartheta e^{i\left(m-n_{e}\right) \vartheta} I_{0}(2 s \cos (\vartheta / 2) \\
& =\frac{1}{\pi I_{0}(2 s)} \int_{0}^{\pi} d \vartheta \cos \left[\left(m-n_{e}\right) \vartheta\right] I_{0}(2 s \cos (\vartheta / 2)
\end{aligned}
$$

for integral (148). As [55,56]

$$
\int_{-\pi / 2}^{\pi / 2} d \beta \cos (2 n \beta) I_{0}(2 a \cos \beta)=\pi I_{n}^{2}(a),
$$

integral (148) indeed gives the same probability,

$$
\left|c_{m}\right|^{2}=\frac{1}{I_{0}(2 s)}\left|I_{m-n_{e}}(s)\right|^{2},
$$

as obtained from Eq. (142).

\section{Thermal states}

Let simple rotators with Hamiltonian (75) be in a heat bath of temperature $T$. Then the density matrix, (62), has the form

$$
\begin{gathered}
\rho=\left(\lambda_{n} \delta_{m n}\right), \quad \lambda_{n}=\frac{e^{-n^{2} \varepsilon \beta}}{Z(\beta)}, \\
\beta=1 /\left(k_{B} T\right), \\
Z(\beta)=\sum_{n \in \mathbb{Z}} e^{-n^{2} \varepsilon \beta} .
\end{gathered}
$$

The partition function, (154), can be expressed in terms of a $\vartheta$ function [57]:

$$
\begin{aligned}
\vartheta_{3}\left(z, q=e^{i \pi \tau}\right) \equiv & \vartheta_{3}(z \mid \tau)=\sum_{n \in \mathbb{Z}} q^{n^{2}} e^{2 n i z} \\
= & 1+2 \sum_{n=1}^{\infty} q^{n^{2}} \cos 2 n z \\
= & \vartheta_{3}(-z, q), \\
& \operatorname{Im}(\tau)>0 .
\end{aligned}
$$

Function (155) is an entire function of $z$, real valued for real and imaginary $z$ if $q$ is real. For real $q$ there are also no zeros on the real and imaginary $z$ axis and $\vartheta_{3}(z, q)$ is positive there.

We now can write

$$
\begin{aligned}
Z(\beta) & =\vartheta_{3}\left(z=0, q=e^{-\varepsilon \beta}\right) \\
& =\vartheta_{3}(z=0 \mid \tau=i \varepsilon \beta / \pi) .
\end{aligned}
$$

For $\varepsilon \beta \gg 1$ (low temperatures) the first order in $q$ in series (156) gives a reasonable approximation:

$$
Z(\beta) \approx 1+2 e^{-\varepsilon \beta}, \quad 1 / Z(\beta) \approx 1-2 e^{-\varepsilon \beta}, \quad \varepsilon \beta \gg 1 .
$$

[For $\varepsilon \approx O(1 \mathrm{eV})$ and $T \approx 300^{\circ} \mathrm{K}$ one has $\varepsilon \beta \approx 40$ ]. A corresponding high-temperature approximation can be obtained 
with the help of Jacobi's famous identity:

$$
\vartheta_{3}(z \mid \tau)=(-i \tau)^{-1 / 2} e^{-i z^{2} /(\pi \tau)} \vartheta_{3}(z / \tau \mid-1 / \tau) .
$$

It yields

$$
Z(\beta)=\left(\frac{\pi}{\varepsilon \beta}\right)^{1 / 2} \vartheta_{3}\left(z=0, q=e^{-\pi^{2} /(\varepsilon \beta)}\right),
$$

which for $\varepsilon \beta \ll 1$ provides the approximation

$$
\begin{gathered}
Z(\beta) \approx\left(\frac{\pi}{\varepsilon \beta}\right)^{1 / 2}\left(1+2 e^{-\pi^{2} /(\varepsilon \beta)}\right), \quad \varepsilon \beta \ll 1, \\
1 / Z(\beta) \approx\left(\frac{\varepsilon \beta}{\pi}\right)^{1 / 2}\left(1-2 e^{-\pi^{2} /(\varepsilon \beta)}\right) .
\end{gathered}
$$

For the Wigner function, (93), we get-according to Eq. (63)-

$$
\begin{aligned}
V_{\rho}(\theta, p) & =\operatorname{tr}[\rho \cdot V(\theta, p)] \\
& =\frac{1}{2 \pi Z(\beta)} \sum_{n \in \mathbb{Z}} e^{-n^{2} \varepsilon \beta} \operatorname{sinc} \pi(p-n) \\
& =\frac{1}{(2 \pi)^{2} Z(\beta)} \int_{-\pi}^{\pi} d \alpha e^{-i p \alpha} \sum_{n \in \mathbb{Z}} e^{-n^{2} \varepsilon \beta} e^{i n \alpha} .
\end{aligned}
$$

This result obviously can be expressed in terms of the $\vartheta$ function, (155), too:

$$
\begin{aligned}
V_{\rho}(\theta, p) & =\operatorname{tr}[\rho \cdot V(\theta, p)] \\
& =\frac{1}{(2 \pi)^{2} Z(\beta)} \int_{-\pi}^{\pi} d \alpha e^{-i p \alpha} \vartheta_{3}\left(\alpha / 2, q=e^{-\varepsilon \beta}\right) \\
& =\frac{1}{2 \pi^{2} Z(\beta)} \int_{0}^{\pi} d \alpha \cos p \alpha \vartheta_{3}\left(\alpha / 2, q=e^{-\varepsilon \beta}\right) .
\end{aligned}
$$

Integrating Eq. (163), or (164), respectively, over $\theta$ gives a factor $2 \pi$; integrating, in addition, over $p$ gives 1 . Multiplying Eq. (163) by sinc $\pi(p-m)$, integrating over $p$, and using relation (54) gives the density matrix elements $\lambda_{n}$ of Eq. (153), multplied by $1 /(2 \pi)$.

In order to gain more insight into the properties of the Wigner function, (164), it helps to look at its low- and hightemperature limits mentioned above. In the low-temperature limit $\varepsilon \beta \gg 1$ we have, for $\vartheta_{3}(\alpha / 2, q=\exp (-\varepsilon \beta))$ at first order of $q$,

$$
\vartheta_{3}\left(\alpha / 2, q=e^{-\varepsilon \beta}\right) \approx 1+2 e^{-\varepsilon \beta} \cos \alpha .
$$

Inserting this into integral (164), observing that [58]

$$
\int_{0}^{\pi} d \alpha \cos \alpha \cos p \alpha=-\frac{\pi}{2} \operatorname{sinc} \pi p\left(\frac{p}{p+1}+\frac{p}{p-1}\right) .
$$

and using approximation (158) yields

$$
V_{\rho}^{(l)}(\theta, p)=\frac{1}{2 \pi} \operatorname{sinc} \pi p\left[1-e^{-\varepsilon \beta}\left(2+\frac{p}{p+1}+\frac{p}{p-1}\right)\right] .
$$

This low-temperature approximation of $V_{\rho}(\theta, p)$ is dominated by the sinc $\pi p$ function in the neighborhood of $p=0$. That function also compensates the poles at $p= \pm 1$ : For, e.g., $p=1+\epsilon$ we have $\lim _{\epsilon \rightarrow 0} \operatorname{sinc} \pi(1+\epsilon) / \epsilon=-1$. Thus, at very low temperatures the wave function, (167), dominantly describes thermally very small angular momenta, as expected.

For the high-temperature approximation we use the relation

$$
\begin{aligned}
& \vartheta_{3}\left(\alpha / 2, q=e^{-\varepsilon \beta}\right) \\
& \quad=\left(\frac{\pi}{\varepsilon \beta}\right)^{1 / 2} e^{-\alpha^{2} /(4 \varepsilon \beta)} \vartheta_{3}\left(z=\frac{i \pi \alpha}{2 \varepsilon \beta}, q=e^{-\pi^{2} /(\varepsilon \beta)}\right),
\end{aligned}
$$

which follows from Jacobi's identity, (159). Due to $\varepsilon \beta \ll$ 1 and the exponent $\pi^{2}$ in $q=\exp \left(-\pi^{2} /(\varepsilon \beta)\right)$, this $q$ is negligible compared to 1 . If the functions $Z(\beta)$ and $\vartheta_{3}$ of Eqs. (160) and (168) are inserted into Eq. (164), their common prefactor drops out. We then get for $V_{\rho}$ the high-temperature approximation

$$
V_{\rho}^{(h)}(\theta, p)=\frac{1}{2 \pi^{2}} \int_{0}^{\pi} d \alpha \cos p \alpha e^{-\alpha^{2} /(4 \varepsilon \beta)} .
$$

As $\varepsilon \beta \ll 1$ the Gaussian under the integral is short-ranged and therefore we can extend the upper limit of the integral from $+\pi$ to $+\infty$ and obtain [59]

$$
V_{\rho}^{(h)}(\theta, p) \approx \frac{\sqrt{\pi \varepsilon \beta}}{2 \pi^{2}} e^{-\varepsilon \beta p^{2}} .
$$

Thus, at high temperatures the Wigner function, (164), becomes a Boltzmann distribution for the classical angular momenta $p$. [Note that $\varepsilon p^{2}$ is the classical counterpart to the quantum mechanical Hamiltonian, (75).] Integrating this $V_{\rho}^{(h)}$ over $p$ gives

$$
\int_{-\infty}^{\infty} d p V_{\rho}^{(h)}(\theta, p)=\frac{1}{2 \pi} .
$$

The denominator $2 \pi$ on the right-hand side is canceled by the final (trivial) integration over $\theta$.

\section{THE CASES $\delta \neq 0$ AND $\hbar \neq 1$}

\section{A. $\delta \neq 0$}

In Ref. [10] a number of physical examples are mentioned for which the parameter $\delta$ in Eqs. (22)-(24) is nonvanishing. Another example with $\delta \neq 0$ is discussed in the preceding subsection. It is, therefore, of interest to indicate the main changes in the principle formulas in Sec. III if $\delta \neq 0$.

Going through the arguments in Sec. II B, above, now using the basis functions, (23), we get, instead of Eqs. (34) and (35),

$$
\begin{aligned}
& V_{m n}^{[\delta]}(\theta, p) \\
& =\frac{1}{(2 \pi)^{2}} e^{i(n-m) \theta} \int_{-\pi}^{\pi} d \vartheta e^{i[(n+m+2 \delta) / 2-p] \vartheta} \\
& =\frac{1}{2 \pi} e^{i(n-m) \theta} \operatorname{sinc} \pi[p-(m+n+2 \delta) / 2] .
\end{aligned}
$$

Relations (36)-(40) now take the form

$$
\begin{gathered}
\int_{-\infty}^{\infty} d p V_{m n}^{[\delta]}(\theta, p)=\frac{1}{2 \pi} e^{i(n-m) \theta}, \\
\int_{-\pi}^{\pi} d \theta V_{m n}^{[\delta]}(\theta, p)=\operatorname{sinc} \pi(p-m-\delta) \delta_{m n}, \\
\int_{-\pi}^{\pi} d \theta \int_{-\infty}^{\infty} d p V_{m n}^{[\delta]}(\theta, p)=\delta_{m n},
\end{gathered}
$$




$$
\begin{gathered}
\operatorname{tr}\left(V_{m n}^{[\delta]}\right)=\frac{1}{2 \pi} \sum_{n \in \mathbb{Z}} \operatorname{sinc} \pi(p-n-\delta)=\frac{1}{2 \pi}, \\
\int_{-\pi}^{\pi} d \theta \int_{-\infty}^{\infty} d p V_{k l}^{[\delta] *}(\theta, p) V_{m n}^{[\delta]}(\theta, p)=\frac{1}{2 \pi} \delta_{k m} \delta_{l n} .
\end{gathered}
$$

Using expansions (24) we get, instead of Eq. (49),

$$
\begin{aligned}
V_{\psi_{2} \psi_{1}}^{[\delta]}(\theta, p) & =\sum_{m, n \in \mathbb{Z}} c_{m}^{(2) *} V_{m n}^{[\delta]}(\theta, p) c_{n}^{(1)} \\
& =\frac{1}{(2 \pi)^{2}} \int_{-\pi}^{\pi} d \vartheta e^{-i p \vartheta} \psi_{2}^{[\delta] *}(\theta-\vartheta / 2) \psi_{1}^{[\delta]}(\theta+\vartheta / 2) \\
& =\left(\psi_{2}^{[\delta]}, V(\theta, p) \psi_{1}^{[\delta]}\right) .
\end{aligned}
$$

Relation (54) is to be replaced with

$$
\int_{-\infty}^{\infty} d p \operatorname{sinc} \pi(m+\delta-p) \operatorname{sinc} \pi(n+\delta-p)=\delta_{m n} .
$$

It is apparently rather obvious how one has to proceed if one passes from a Hilbert space with $\delta=0$ to one with $\delta \neq 0$, e.g., in Secs. IV A and IV B.

\section{B. $\hbar \neq 1$}

In the sections above we have put $\hbar=1$. Making $\hbar$ explicit again in all the formulas is easier here than in the $(q, p)$ case, because the basic variable $\theta$ is dimensionless and only the canonically conjugate angular momentum $p$, which has the dimension (action) has to be rescaled.

In order to reintroduce $\hbar$ into the above formulas the following two replacements are necessary: First, the angular momentum operator $L$ of Eq. (25) has to be rescaled:

$$
L=\frac{1}{i} \partial_{\varphi} \rightarrow \hat{L}=\frac{\hbar}{i} \partial_{\varphi}
$$

Note that the basis functions, (23), remain unchanged and are now eigenfunctions of $\hat{L}$ with eigenvalues $\hbar n$. In addition, the classical phase-space variable $p$, which we have treated as dimensionless above, has to be replaced with $p / \hbar$ if $p$ is now interpreted as a variable with the dimension (action).

As an example, consider the sinc function of Eq. (1):

$$
\begin{aligned}
\operatorname{sinc} \pi(p-m) & \rightarrow \operatorname{sinc}[\pi(p-\hbar m) / \hbar] \\
& =\hbar \frac{\sin [\pi(p-m \hbar) / \hbar]}{\pi(p-\hbar m)} .
\end{aligned}
$$

Thus, the sinc function, (182), is dimensionless. So are the matrix $V(\theta, p)$ and the wave functions, (48). As

$$
\lim _{\epsilon \rightarrow 0} \frac{\sin (\pi x / \epsilon)}{\pi x}=\delta(x),
$$

the associated classical limit of the rescaled expression, (182), can be obtained as

$$
\lim _{\hbar \rightarrow 0} \frac{1}{\hbar} \operatorname{sinc}[\pi(p-\hbar m) / \hbar]=\delta(p-\hbar m), \quad \hbar m=\text { const. }
$$

In Sec. III C the time derivatives $\partial_{t}$ have to be replaced with $\hbar \partial_{t}$.

\section{REMARKS}

\section{A. Dirac notation}

Throughout the preceding text I have avoided the use of the widespread Dirac notion of "bra", "ket," etc., for describing quantum mechanical states and operators. The reason is (see, e.g., Ref. [10]) that there is no mathematically well-defined angle operator $\hat{\varphi}$ with eigenfunctions $|\varphi\rangle$ such that

$$
\hat{\varphi}|\varphi\rangle=\varphi|\varphi\rangle .
$$

The use of such mathematically nonexistent objects might be helpful heuristically if one is appropriately careful: Here the formal objects $|\varphi\rangle$ only make sense in the combination

$$
\langle\varphi \mid n\rangle=e^{i n \varphi}=e_{n}(\varphi), \quad L|n\rangle=n|n\rangle,
$$

where $L$ is the well-defined angular momentum operator. Armed with this mental reservation one may write for the operator $V$ in Eq. (34)

$$
\tilde{V}(\theta, p)=\frac{1}{(2 \pi)^{2}} \int_{-\pi}^{\pi} d \vartheta e^{-i p \vartheta}|\theta-\vartheta / 2\rangle\langle\theta+\vartheta / 2|,
$$

the matrix elements $\tilde{V}_{m n}=\langle m|\tilde{V}| n\rangle$ of which are the same as those in Eq. (34). The Wigner function in Eq. (81) may be written as

$$
V_{\psi}(\theta, p)=\tilde{V}_{\psi}(\theta, p)=\langle\psi|\tilde{V}(\theta, p)| \psi\rangle,
$$

where $|\psi\rangle$ is to be considered as an expansion in terms of the eigenstates $|n\rangle$.

Another interesting example is (see Sec. III B)

$$
\begin{aligned}
\operatorname{tr}[V(\theta, p) \cdot A] & =\operatorname{tr}[\tilde{V}(\theta, p) \cdot A] \\
& =\frac{1}{(2 \pi)^{2}} \int_{-\pi}^{\pi} d \vartheta e^{-i p \vartheta}\langle\theta+\vartheta / 2|A| \theta-\vartheta / 2\rangle
\end{aligned}
$$

Inserting the completeness relations $\sum_{m \in \mathbb{Z}}|m\rangle\langle m|=\mathbf{1}$ and $\sum_{n \in \mathbb{Z}}|n\rangle\langle n|=\mathbf{1}$ before and after the operator $A$ in the last expression leads back to the results in Sec. III B.

\section{B. A possible generalization and some related work}

The approach discussed above for phase spaces of the topological type $S^{1} \times \mathbb{R}$ can be generalized, e.g., to a free rigid body in three dimensions with one point fixed. Its configuration space can be identified with the group $\mathrm{SO}(3)[60,61]$. Its twofold covering $\mathrm{SU}(2)$ has the topology of $S^{3}$. The associated phase space $S^{3} \times \mathbb{R}^{3}$ can be quantized in terms of unitary representations of the Euclidean group E(4) [62].

At a late stage of the present investigations I became aware of the work by Leaf [63] in which the operator $\Delta(q, p)$ plays a role with respect to the $(q, p)$ Wigner function which corresponds to that of the operator/matrix $V(\theta, p)$ in the discussions above. Leaf's approach was used by de Groot and Suttorp in their textbook [43].

A Wigner-Moyal function on the circle very similar in structure to the one in Eq. (49) is given in Refs. [64] and [15]. There, however, the classical angular momentum is treated as 
discontinuous, contrary to its properties in its classical phase space and its treatment in the present paper.

\section{ACKNOWLEDGMENTS}

I am again very grateful to the DESY Theory Group for its continuous generous hospitality since my retirement from the
Institute for Theoretical Physics of the RWTH Aachen. I thank Krzysztof Kowalski for drawing my attention first to Ref. [64] and then to Ref. [15]. Finally, I thank Hartmann Römer for a critical reading of the manuscript and the suggestion to include thermal states among the examples in Sec. IV.
[1] E. Wigner, On the quantum correction for thermodynamic equilibrium, Phys. Rev. 40, 749 (1932).

[2] M. Hillery, R. F. O'Connell, M. O. Scully, and E. P. Wigner, Distribution functions in physics: Fundamentals, Phys. Rep. 106, 121 (1984).

[3] U. Leonhardt, Measuring the Quantum State of Light, Cambridge Studies in Modern Optics, Vol. 22 (Cambridge University Press, Cambridge, UK, 1997).

[4] W. P. Schleich, Quantum Optics in Phase Space (Wiley-VCH, Berlin, 2001).

[5] H. Römer, Theoretical Optics: An Introduction, 2nd ed. (Wiley$\mathrm{VCH}$, Weinheim, 2009).

[6] U. Leonhardt, Essential Quantum Optics: From Quantum Measurements to Black Holes (Cambridge University Press, Cambridge, UK, 2010).

[7] G. S. Agarwal, Quantum Optics (Cambridge University Press, Cambridge, UK, 2013).

[8] K. Gröchenig, Foundations of Time-Frequency Analysis (Springer Science + Business Media, New York, 2001).

[9] L. Cohen, The Weyl Operator and Its Generalization, PseudoDifferential Operators, Theory and Applications, Vol. 9 (Birkhäuser, Springer, Basel, 2013).

[10] H. A. Kastrup, Quantization of the canonically conjugate pair angle and orbital angular momentum, Phys. Rev. A 73, 052104 (2006). (Here Appendix A. Compared to this Ref. [10] the present follow-up paper has some minor changes in notation.)

[11] M. V. Berry, Semi-classical mechanics in phase space: A study of Wigner's function, Philos. Trans. Roy. Soc. London A 287, 237 (1977).

[12] N. Mukunda, Wigner distribution for angle coordinates in quantum mechanics, Am. J. Phys. 47, 182 (1979).

[13] I. Rigas, L. L. Sánchez-Soto, A. B. Klimov, J. Řeháček, and Z. Hradil, Orbital angular momentum in phase space, Ann. Phys. (NY) 326, 426 (2011).

[14] M. Przanowski, P. Brzykcy, and J. Tosiek, From the Weyl quantization of a particle on the circle to number-phase Wigner functions, Ann. Phys. (NY) 351, 919 (2014).

[15] N. Mukunda, G. Marmo, A. Zampini, S. Chaturvedi, and R. Simon, Wigner-Weyl isomorphism for quantum mechanics on Lie groups, J. Math. Phys. 46, 012106 (2005).

[16] C. Fronsdal, Some ideas about quantization, Rep. Math. Phys. 15, 111 (1979).

[17] M. Gadella, M. A. Martin, L. M. Nieto, and M. A. del Olmo, The Stratonovich-Weyl correspondence for one-dimensional kinematical groups, J. Math. Phys. 32, 1182 (1991).

[18] O. Arratia and M. A. del Olmo, Moyal quantization on the cylinder, Rep. Math. Phys. 40, 149 (1997).

[19] C. J. Isham, Topological and global aspects of quantum theory, in Relativity, Groups and Topology II. Les Houches Session XL,
1983, edited by B. S. DeWitt and R. Stora (North-Holland, Amsterdam, 1984), p. 1059 and, especially, pp. 1170-1176, $1224-1226$.

[20] K. B. Wolf, Wigner distribution function for paraxial polychromatic optics, Opt. Commun. 132, 343 (1996).

[21] L. M. Nieto, N. M. Atakishiyev, S. M. Chumakov, and K. B. Wolf, Wigner distribution function for Euclidean systems, J. Phys. A: Math. Gen. 31, 3875 (1998).

[22] S. T. Ali, N. M. Atakishiyev, S. M. Chumakov, and K. B. Wolf, The Wigner function for general Lie groups and the wavelet transform, Ann. Henri Poincaré 1, 685 (2000).

[23] J. E. Moyal, Quantum mechanics as a statistical theory, Proc. Cambr. Philos. Soc. 45, 99 (1949).

[24] G. B. Folland, Harmonic Analysis in Phase Space, Ann. Math. Studies, Vol. 122 (Princeton University Press, Princeton, NJ, 1989), Chaps. 1 and 2.

[25] M. de Gosson, Symplectic Geometry and Quantum Mechanics, Operator Theory: Advances and Applications, Vol. 166 (Birkhäuser, Basel, 2006), parts II and III.

[26] E. T. Whittaker, On the functions which are represented by the expansions of the interpolation-theory, Proc. Roy. Soc. Edinburgh 35, 181 (1915).

[27] J. McNamee, F. Stenger, and E. L. Whitney, Whittaker's cardinal function in retrospect, Math. Comput. 25, 141 (1971).

[28] F. Stenger, Numerical methods based on Whittaker cardinal, or sinc functions, SIAM Rev. 23, 165 (1981).

[29] P. L. Butzer, A survey of the Whittaker-Shannon sampling theorem and some of its extensions, J. Math. Res. Expos. 3, 185 (1983).

[30] J. R. Higgins, Five short stories about the cardinal series, Bull. (N.S.) Am. Math. Soc. 12, 45 (1985).

[31] F. Stenger, Numerical Methods Based on Sinc and Analytic Functions, Springer Series in Computational Mathematics, Vol. 20 (Springer-Verlag, New York, 1993).

[32] F. Stenger, Handbook of Sinc Numerical Methods, Chapman \& Hall/CRC Numerical Analysis and Scientific Computing (CRC Press Taylor \& Francis Group, Boca Raton, FL, 2011).

[33] M. Vetterli, J. Kovačević, and V. K. Goyal, Foundations of Signal Processing (Cambridge University Press, Cambridge, UK, 2014).

[34] Y. C. Eldar, Sampling Theory (Cambridge University Press, Cambridge, UK, 2015).

[35] W. H. Louisell, Amplitude and phase uncertainty relations, Phys. Lett. 7, 60 (1963).

[36] G. W. Mackey, The Mathematical Foundations of Quantum Mechanics (W. A. Benjamin, New York, 1963), p. 103.

[37] N. I. Vilenkin, Special Functions and the Theory of Group Representations, Translations of Mathematical Monographs, Vol. 22 (Am. Math. Soc., Providence, RI, 1968), Chap. IV. 
[38] M. Sugiura, Unitary Representations and Harmonic Analysis: An Introduction, North-Holland Mathematical Library, Vol. 44 (Elsevier, Amsterdam, 1990), Chap. IV.

[39] G. S. Chirikjian and A. B. Kyatkin, Engineering Applications of Noncommutative Harmonic Analysis, with Emphasis on Rotation and Motion Groups (CRC Press, Boca Raton, FL, 2000), p. 151, (Of general interest for the present paper is especially Chap. 10).

[40] E. T. Whittaker and G. N. Watson, A Course of Modern Analysis, 4th ed. (Cambridge University Press, Cambridge, UK, 1969), p. 362.

[41] P. M. Morse and H. Feshbach, Methods of Theoretical Physics, Vol. I, International Series in Pure and Applied Physics (McGraw-Hill, New York, 1953), p. 766, formula (6.3.62).

[42] A. P. Prudnikov, Y. A. Brychkov, and O. I. Marichev, Integrals and Series, Vol. 1 (Gordon and Breach Science, New York, 1986), p. 727, formula 13.6.

[43] S. R. de Groot and L. G. Suttorp, Foundations of Electrodynamics (North-Holland, Amsterdam, 1972), Chap. VI. (The representation here relies on Ref. [63].)

[44] G. H. Hardy, Notes on special systems of orthogonal functions (IV): The orthogonal functions of Whittaker's series, Proc. Cambr. Philos. Soc. 37, 331 (1941), reprinted in Collected Papers of G. H. Hardy, Vol. III (Clarendon Press, Oxford, UK, 1969), p. 466.

[45] O. Christensen, Frames and Bases, an Introductory Course, Applied and Numerical Harmonic Analysis (Birkhäuser, Boston, 2008), Chap. 3.8.

[46] See, e.g., Ref. [4], Chap. 4, or Ref. [7], Chap. 1.8.

[47] J. Řeháček, Z. Bouchal, R. Čelechovský, Z. Hradil, and L. L. Sánchez-Soto, Experimental test of uncertainty relations for quantum mechanics on a circle, Phys. Rev. A 77, 032110 (2008).

[48] H. P. Robertson, The uncertainty principle, Phys. Rev. 34, 163 (1929).

[49] E. Schrödinger, Zum Heisenbergschen Unschärfeprinzip, Sitzungsber. Preuss. Akad. Wiss., Phys.-math. Klasse 19, 296
(1930), reprinted in E. Schrödinger, Collected Papers, Vol. 3 (Braunschweig, Wien, 1984), p. 348.

[50] R. Jackiw, Minimum uncertainty product, number-phase uncertainty product, and coherent states, J. Math. Phys. 9, 339 (1968).

[51] C. Forbes, M. Evans, and N. Hastings, Statistical Distributions, 4th ed. (John Wiley \& Sons, Hoboken, NJ, 2011), Chap. 45.

[52] K. V. Mardia and P. E. Jupp, Directional Statistics, Wiley Series in Probability and Statistics (John Wiley \& Sons, Chichester, UK, 2000), especially subsec. 3.5.4.

[53] G. N. Watson, A Treatise on the Theory of Bessel Functions, 2nd ed. (Cambridge University Press, Cambridge, UK, 1966), p. 181, formula (4).

[54] See, e.g., Ref. [53], p. 698.

[55] Edited by A. Erdélyi, Tables of Integral Transforms, Vol. I (McGraw-Hill, New York, 1954), p. 59, formula (61).

[56] I. S. Gradshteyn and I. M. Ryzhik, Table of Integrals, Series and Products, 4th ed. (Academic Press, New York, 1965), p. 738, formula $6.681,3$. (The formula number is the same in later editions.)

[57] For the literature on the $\vartheta$ functions, see Appendix C in Ref. [10].

[58] Ref. [42], p. 403, formula 41.

[59] Ref. [56], p. 480, formula 3.896, 4.

[60] See, e.g., Ref. [39], Chaps. 5 and 6.

[61] J. E. Marsden and T. S. Ratiu, Introduction to Mechanics and Symmetry, 2nd ed. (Springer-Verlag, New York, 2003), Chap. 15.

[62] Ref. [19], example 4.9 (p. 1194). (Regarding irreducible unitary representations of Euclidean groups $E(n)$, see, e.g., Chap. XI in Ref. [37] and Chap. 10 in Ref. [39].)

[63] B. Leaf, Weyl transformation and the classical limit of quantum mechanics, and Weyl transform in nonrelativistic quantum dynamics, J. Math. Phys. 9, 65 (1968); 9, 769 (1968).

[64] M. A. Alonso, G. S. Pogosyan, and K. B. Wolf, Wigner functions for curved spaces. II. On spheres, J. Math. Phys. 44, 1472 (2003). (Here Sec. IV A.) 